\title{
Effectiveness of Antipsychotics on Delirium in Elderly Patients
}

Miji Lee ${ }^{1}$, Taeseung Kim¹, Sohyun Ahn' ${ }^{1}$ and Jeong Lan Kim ${ }^{12^{*}}$

${ }^{1}$ Department of Psychiatry, Chungnam National University Hospital, Daejeon, Republic of Korea

${ }^{2}$ Department of Psychiatry, School of Medicine, Chungnam National University, Daejeon, Republic of Korea

\begin{abstract}
Study background: Delirium is common in older adults, but the pharmacological treatment of delirium in the elderly has not been established. This study aimed to compare the efficacy of three atypical antipsychotics (amisulpride, quetiapine, and risperidone) for the treatment of delirium in patients aged $>65$ years.

Methods: Psychiatrists examined subjects aged $>65$ years referred to the consultation-liaison psychiatry service for at baseline, day 3 , and day 7 . Totally, 129 subjects were enrolled and assigned to receive either amisulpride $(n=44)$, quetiapine $(n=44)$, risperidone $(n=21)$, or not to receive any antipsychotic medications $(n=20)$. We examined the efficacy of each medication using the Korean version of the Delirium Rating Scale-Revised-98, which investigates the severity of delirium episodes. We then evaluated the cognitive function of subjects using the Mini-Mental Status Examination.
\end{abstract}

Results: At baseline, no significant differences were found among the four groups with regard to mean Korean version of the Delirium Rating Scale-Revised-98 or Mini-Mental Status Examination scores. In all groups, including the non-antipsychotic group, the Korean version of the Delirium Rating Scale-Revised-98 score decreased and Mini-Mental Status Examination score increased a week after the delirium diagnosis. There were no significant differences in improved Korean version of the Delirium Rating Scale-Revised-98 or Mini-Mental Status Examination scores among these four groups.

Conclusion: Compared to subjects taking atypical antipsychotics, subjects not taking any antipsychotic medications equally improved their delirium symptoms and cognition. For hospitalized older adults with acute delirium, the data suggests that atypical antipsychotic treatment is ineffective for improving delirium outcomes.

Keywords: Elderly; Delirium; Amisulpride; Quetiapine; Risperidone

\section{Introduction}

Delirium is a complex neuropsychiatric disorder that occurs commonly among patients in all health care settings, but especially among the elderly [1]. It is characterized by an acute decline in consciousness and cognition, and it causes impairment in attention. In general hospital admissions, delirium prevalence ranges from $6 \%$ to $56 \%$ [2], with higher prevalence associated with increasing age [3] and illness severity [4]. Recent evidence indicates that delirium in elderly patients is associated with increased risk of death, institutionalization, and dementia, independent of age, sex, comorbid illness, illness severity, and presence of dementia at baseline [5]. Because delirium is common in elderly patients and associated with poor outcomes, it is important to predict, prevent, diagnose, and treat affected patients.

First, delirium treatment requires elucidation and correction of causative factors. However, it is also necessary to treat delirium symptoms at bedside. Several classes of medications have been used to treat delirium symptoms, including atypical and typical antipsychotic medications, cholinesterase inhibitors, antidepressants, benzodiazepines, and gabapentin [6]. Pharmacological interventions are often used for treating delirium, but the Food and Drug Administration has yet to approve any of them. Indeed, there have been few randomized, controlled trials on acute drug treatments for delirium. The neurophysiological mechanisms of delirium are poorly understood, but evidence suggests that pre-existing deficiencies in cholinergic neurotransmission may be a contributing factor [7].

Among pharmacological interventions, it is widely accepted that antipsychotic medication is the first-line pharmacotherapy for delirium, except in case of sedative use or alcohol withdrawal. Physicians have used typical antipsychotics, primarily haloperidol, to control delirium symptoms, which might compromise the safety of patients or caregivers [8]. Because typical antipsychotics are associated with more drug-induced side effects such as extrapyramidal symptoms, emerging literature focuses on the use of atypical antipsychotics for the pharmacological management of acute delirium. A number of researchers have reported that atypical antipsychotics, including olanzapine, quetiapine and risperidone, might be as effective as haloperidol in treating delirium [9].

Although previous trials have reported no significant differences in efficacy between haloperidol and atypical antipsychotics in delirium treatment [8-11], the reported data are not sufficient to conclude that the efficacy of various atypical antipsychotics is superior in the treatment of delirium. Therefore, this study aimed to compare the efficacy of three antipsychotic medications (amisulpride, quetiapine, and risperidone) for treating delirium in patients with medical illnesses aged $>65$ years in an inpatient setting. The study also includes patients not using antipsychotics as a basis for comparison.

\section{Materials and Methods}

\section{Subjects}

Patients aged $>65$ years were recruited from a tertiary care university hospital. The participants were referred to the consultation-

*Corresponding author: Jeong Lan Kim, Department of Psychiatry, Chungnam National University Hospital, Daejeon, Republic of Korea, Tel: +82422807287; Fax: +82422807886; E-mail: kimjl@cnu.ac.kr

Received: May 22, 2017; Accepted: June 06, 2017; Published: June 08, 2017

Citation: Lee M, Kim T, Ahn S, Kim JL (2017) Effectiveness of Antipsychotics on Delirium in Elderly Patients. J Psychiatry 20: 411. doi:10.4172/2378-5756.1000411

Copyright: ( 2017 Lee M, et al. This is an open-access article distributed under the terms of the Creative Commons Attribution License, which permits unrestricted use, distribution, and reproduction in any medium, provided the original author and source are credited 
liaison psychiatry service. A psychiatrist made the diagnoses according to the diagnostic criteria of the Diagnostic and Statistical Manual of Mental Disorders 4th edition (DSM-IV) [12]. The procedures and rationale for this study were explained to obtain informed consent from the patient or the proxy decision maker. Patients were excluded if they refused evaluation procedures or withdrew their consent during the following examinations. This study was approved by the Institutional Review Board and Ethics Committee of Chungnam National University Hospital

\section{Measures}

The Delirium Etiology Checklist (DEC): DEC assesses the risk factors for delirium [13]. Using DEC, raters placed the standardized attribution of etiology into 12 categories of causation according to all available clinical information. Each rater used a 5-point scale for degrees of attribution to the delirium episode, ranging from "ruled out/not present/not relevant" to "definite cause" as follows: drug intoxication/ drug withdrawal; metabolic/endocrine disturbance; traumatic brain injury; seizures; infection (intracranial); infection (systemic); neoplasm (intracranial); neoplasm (systemic); cerebrovascular; organ insufficiency; other central nervous system (CNS); and other systemic causes. This system allows for the documentation of multiple potential causes of delirium. DEC provides more specific and relevant information regarding delirium than a list of current medical conditions.

The Korean version of the Delirium Rating Scale-Revised-98 (K-DRS-98): DRS-R98 was created to compensate for the shortcomings of DRS [14], especially for use in phenomenological and longitudinal research. DRS-R98 is composed of two sections. The first section is a severity scale, consisting of 13 items that are scored from 0 to 3 according to a Likert scale anchored by descriptions of phenomenology. The second section consists of three diagnostic items that are scored from 0 to 2 or 0 to 3 . The severity scale scores range from 0 to 39 , with higher scores indicating more severe delirium. A cutoff score of $\geq 15$ is consistent with a diagnosis of delirium. Even in mixed neuropsychiatric populations, the scale has strong inter-rater reliability, validity, sensitivity, and specificity for distinguishing delirium, including dementia, depression, and schizophrenia.

This study employs the Korean version of the DRS-98 (K-DRS-98). This scale has shown high internal consistency with a Cronbach's alpha coefficient of 0.87 for the total scale and 0.86 for the severity scales. Inter- rater reliability ( $\kappa=0.9$ for severity items) is also high. The suggested optimal cutoff point for delirium diagnosis is a severity score of 16 and a total score of 21.5 [15].

Mini-Mental State Examination (MMSE): MMSE is a simplified scored form of the cognitive mental status examination known as the Mini-Mental State (MMS), which includes 11 questions. This 30-point instrument assesses cognitive impairment and covers five broad areas of cognitive function: orientation (10 points), registration (3 points), attention and calculation (5 points), recall (3 points), and language (9 points). It has been validated in patients with dementia syndrome, affective disorder, mania, schizophrenia, and personality disorders [16].

\section{Procedures}

We designed a 7-day, prospective, comparative clinical observational study. On day 1 of diagnosis, all subjects who were eligible for the study were examined by psychiatrists using the K-DRS-98, MMSE, and DEC. Psychiatrists rated the K-DRS-98 and MMSE on the days 3 and 7 following the diagnosis date. Each patient was randomly assigned to receive one of the following three drugs-amisulpride, quetiapine, and risperidone-with a flexible dosing schedule, or not to receive any of them (non-antipsychotic group). To obtain data for proper rating, available information was reviewed, including medical records, nursing records, and interviews with caregivers or family members. The K-DRS-98 rating was based on a $24 \mathrm{~h}$ period because of the fluctuating nature of delirium symptoms. After day 7 of diagnosis, we reviewed the subjects' medical records regarding prescribed medications and found out that some patients did not receive the recommended antipsychotic medications despite the consultation, on the basis of the referring clinician's judgment. We classified these cases in to the nonantipsychotic group.

\section{Data Analysis}

Data were analyzed using the Statistical Package for Social Sciences, version 21 (SPSS; Chicago, IL). Descriptive analysis was performed in the form of percentages, means, and standard deviations. Chi-square test or Fisher's exact test was used to compare the categorical variables. One-way analysis of variance was conducted to analyze the continuous variables. Statistical significance was set at an alpha level of 0.05 .

\section{Results}

\section{Study population}

A total of 129 subjects were enrolled and divided into four groups: the amisulpride group $(n=44)$, the quetiapine group $(n=44)$, the risperidone group $(n=21)$, and the non-antipsychotic group $(n=20)$. The mean age of patients was $76.2 \pm 6.6$ years. There were 84 men (65.1\%) and 45 women (34.9\%). Ninety-one patients (70.5\%) completed assessments on day 7 of diagnosis.

Table 1 summarizes the demographic data for each group. The mean age and gender for all four groups showed no significant difference ( $\mathrm{p}=0.334$ and 0.331 , respectively). The amisulpride group used the medication at mean doses of $50 \mathrm{mg} /$ day. The quetiapine and risperidone groups took the medication at mean doses of $44.4 \mathrm{mg} / \mathrm{day}$ and $0.8 \mathrm{mg} / \mathrm{day}$, respectively. There were no significant differences in dosage of the prescribed antipsychotic medications when converted to the equivalent haloperidol dose (amisulpride $50.0 \mathrm{mg} /$ day, quetiapine $57.8 \pm 19.2 \mathrm{mg} /$ day, risperidone $55.6 \pm 16.1 \mathrm{mg} /$ day; $\mathrm{r}=0.035$ ) [17]. The number of subjects completing assessments decreased from time to time, but dropout rates were similar in all four groups.

\section{Associations with etiologies of delirium}

With regards to the contributing etiological factors of delirium, the mean number of all possible causes per patient was about two $(2.1 \pm 0.9)$. Half of the patients had three $(n=54,41.9 \%)$ contributing causes of delirium. Table 2 illustrates the primary etiologies underlying the delirium of study subjects. For this study's analysis, the ratings of "definite cause," "likely cause," and "cause present and possibly contributory" were combined and considered as present; the ratings of "present but not contributory" and "ruled out/not present/not relevant" were considered as absent. The most common underlying etiology in the groups was a systemic neoplasm $(n=34,26.4 \%)$, followed by systemic infection $(\mathrm{n}=23,17.8 \%)$.

\section{Changes in delirium symptoms with time}

The baseline K-DRS-98 total score was $27.2 \pm 8.0,28.1 \pm 6.5,26.1 \pm$ 7.1 , and $26.8 \pm 9.9$ in the amisulpride, quetiapine, risperidone, and nonantipsychotic groups. On day 1 of enrollment, there were no significant differences in the mean K-DRS-98 total scores among the four groups $(\mathrm{p}=0.846)$ 
Citation: Lee M, Kim T, Ahn S, Kim JL (2017) Effectiveness of Antipsychotics on Delirium in Elderly Patients. J Psychiatry 20: 411. doi:10.4172/23785756.1000411

Page 3 of 6

\begin{tabular}{|c|c|c|c|c|c|c|}
\hline Characteristics & $\begin{array}{l}\text { Amisulpride group } \\
\mathrm{n}=44\end{array}$ & $\begin{array}{l}\text { Quetiapine group } \\
n=44\end{array}$ & $\begin{array}{l}\text { Risperidone group } \\
\mathrm{n}=21\end{array}$ & $\begin{array}{c}\text { Non-antipsychotic } \\
\text { group } \\
n=20\end{array}$ & $\begin{array}{c}\text { Total } \\
n=129\end{array}$ & Sig. \\
\hline Age, year & $75.1 \pm 5.8$ & $77.6 \pm 7.1$ & $75.6 \pm 7.0$ & $75.9 \pm 6.3$ & $76.2 \pm 6.6$ & 0.334 \\
\hline Gender, male & $26(59.1)$ & $30(68.2)$ & $12(57.1)$ & $16(80)$ & $84(65.1)$ & 0.331 \\
\hline Dose, mg/day & $50.0 \pm 0.0$ & $44.4 \pm 14.7$ & $0.8 \pm 0.2$ & & & \\
\hline \multicolumn{7}{|c|}{ Number of subjects assessed } \\
\hline Baseline & $44(100.0)$ & $44(100.0)$ & $21(100.0)$ & $20(100.0)$ & $129(100.0)$ & \\
\hline Day 3 & $42(95.5)$ & $42(95.5)$ & $20(95.2)$ & $18(90.0)$ & $122(94.6)$ & \\
\hline Day 7 & $30(68.2)$ & $30(68.2)$ & $17(81.0)$ & $14(70.0)$ & $91(70.5)$ & \\
\hline
\end{tabular}

Table 1: Group comparisons of demographic characteristics, medications, and number of subjects assessed.

\begin{tabular}{|c|c|c|c|c|c|}
\hline Etiologies & $\begin{array}{l}\text { Amisulpride group } \\
\mathrm{n}=44\end{array}$ & $\begin{array}{l}\text { Quetiapine group } \\
\qquad n=44\end{array}$ & $\begin{array}{l}\text { Risperidone group } \\
n=21\end{array}$ & $\begin{array}{l}\text { Non-antipsychotic group } \\
n=20\end{array}$ & $\begin{array}{c}\text { Total } \\
n=129 \\
(100 \%)\end{array}$ \\
\hline Drug intoxication/drug withdrawal & 0 & 0 & 0 & 0 & 0 \\
\hline Metabolic/endocrine disturbance & 1 & 1 & 0 & 1 & $3(2.3 \%)$ \\
\hline Traumatic brain injury & 4 & 4 & 1 & 0 & $9(7.0 \%)$ \\
\hline Seizures & 0 & 0 & 0 & 0 & 0 \\
\hline Infection (intracranial) & 0 & 0 & 0 & 0 & 0 \\
\hline Infection (systemic) & 12 & 7 & 1 & 3 & $23(17.8 \%)$ \\
\hline Neoplasm (intracranial) & 4 & 1 & 0 & 1 & $6(4.7 \%)$ \\
\hline Neoplasm (systemic) & 8 & 13 & 6 & 7 & $34(26.4 \%)$ \\
\hline Cerebrovascular & 3 & 4 & 3 & 2 & $12(9.3 \%)$ \\
\hline Organ insufficiency & 0 & 1 & 1 & 2 & $4(3.1 \%)$ \\
\hline Other CNS & 1 & 1 & 0 & 0 & $2(6.9 \%)$ \\
\hline Other systemic & 11 & 12 & 9 & 4 & $36(27.9 \%)$ \\
\hline
\end{tabular}

Table 2: Primary etiologies underlying delirium in study population.

\begin{tabular}{|c|c|c|c|c|c|c|}
\hline Efficacy measure & Amisulpride group $n=30$ & $\begin{array}{l}\text { Quetiapine group } \\
\qquad \mathrm{n}=30\end{array}$ & $\underset{n=17}{\text { Risperidone group }}$ & $\begin{array}{c}\text { Non-antipsychotic group } \\
n=14\end{array}$ & Totaln $=91$ & Sig. \\
\hline \multicolumn{2}{|c|}{ K-DRS-98, severity score } & & & & & \\
\hline Baseline & $21.6 \pm 7.4$ & $22.6 \pm 6.4$ & $20.3 \pm 6.4$ & $21.1 \pm 8.9$ & $21.6 \pm 7.1$ & 0.739 \\
\hline Day 3 & $15.8 \pm 8.3$ & $15.6 \pm 5.0$ & $15.4 \pm 6.1$ & $14.4 \pm 8.0$ & $15.4 \pm 7.0$ & 0.932 \\
\hline Day 7 & $11.6 \pm 6.8$ & $10.7 \pm 5.5$ & $12.8 \pm 7.0$ & $12.8 \pm 9.2$ & $11.7 \pm 6.8$ & 0.695 \\
\hline \multicolumn{2}{|c|}{ K-DRS-98, total score } & & & & & \\
\hline Baseline & $27.2 \pm 8.0$ & $28.1 \pm 6.5$ & $26.1 \pm 7.1$ & $26.8 \pm 9.9$ & $27.3 \pm 7.6$ & 0.846 \\
\hline Day 3 & $21.0 \pm 9.5$ & $21.2 \pm 5.8$ & $21.1 \pm 6.4$ & $19.6 \pm 9.1$ & $20.9 \pm 7.7$ & 0.933 \\
\hline Day 7 & $16.4 \pm 8.0$ & $15.6 \pm 6.8$ & $17.6 \pm 8.8$ & $17.6 \pm 10.4$ & $16.5 \pm 8.1$ & 0.815 \\
\hline \multicolumn{2}{|c|}{ MMSE score } & & & & & \\
\hline Baseline & $9.8 \pm 8.9$ & $8.3 \pm 6.4$ & $11.9 \pm 5.7$ & $9.8 \pm 8.0$ & $9.7 \pm 7.4$ & 0.485 \\
\hline Day 3 & $12.7 \pm 8.2$ & $10.3 \pm 7.2$ & $11.9 \pm 5.6$ & $13.4 \pm 5.7$ & $11.9 \pm 7.0$ & 0.512 \\
\hline Day 7 & $15.0 \pm 7.7$ & $13.7 \pm 6.9$ & $12.5 \pm 6.6$ & $16.9 \pm 8.1$ & $14.3 \pm 7.3$ & 0.483 \\
\hline
\end{tabular}

Note: K-DRS-98: Korean version of the Delirium Rating Scale-Revised-98; MMSE: Mini-Mental Status Examination

Table 3: Group comparisons of serial changes in K-DRS-98 and MMSE scores.

As shown in Table 3, the K-DRS-98 severity score and total score for all four groups decreased in the subsequent days, which indicates recovery from delirium symptoms. There were no significant differences in the improvement of K-DRS-98 scores among the three different antipsychotic treatment groups and the non-antipsychotic group. The K-DRS-98 severity score and total score showed no difference on day 7 , even in patients who were not prescribed any antipsychotic medications $(\mathrm{p}=0.695)$.

The group differences for each subscale score of the K-DRS-98 showed no significant changes on days 3 and 7 of diagnosis. There were also no significant differences on days 3 and 7 of diagnosis between the four groups with regard to temporal onset of symptoms, fluctuation of symptom severity, and physical disorder item mean score at baseline.

\section{Changes in cognitive function with time}

At the baseline, the mean score of MMSE was 9.7, which demonstrates that subjects' cognitive functions were seriously impaired. In all groups, the baseline MMSE score showed no significant difference $(p=0.739)$. After day 7, the MMSE score for the four groups increased significantly (an average of 4.6 points), representing a recovery of cognitive function. With regard to improvement in the MMSE score, differences were not significant between any of the groups $(p=0.483)$.

\section{Discussion and Conclusion}

This study is an open-labeled trial of atypical antipsychotics for delirium treatment in hospitalized elderly patients. Although previous studies regarding the efficacy of antipsychotic medication for 
delirium treatment have shown that there are no significant differences between typical and atypical antipsychotics [18], the data remained insufficient to compare the efficacy of various atypical antipsychotics in treating delirium, especially in elderly subjects. Moreover, delirium management includes ensuring safety in the environment or supportive interventions, identifying and treating the cause(s) of delirium, and enhancing the patient's functioning. To our knowledge, this trial is the first to directly compare the efficacy and cognitive outcomes of three atypical antipsychotics, as well as the use of no antipsychotics, in the acute treatment of delirium in a naturalistic environment focusing on elderly inpatients.

In this trial, among 129 subjects who were admitted at tertiary care university hospital, the most common etiologies associated with delirium were systemic neoplasm and systemic infection. There were 34 subjects whose etiology of delirium was primarily systemic neoplasm, and they were treated with quetiapine $(n=13)$, amisulpride $(n=8)$, risperidone $(n=6)$, or not treated with any antipsychotics $(n=7)$. There were 23 patients whose etiology of delirium was primarily systemic infection, and they were treated with amisulpride $(n=12)$, quetiapine $(n=7)$, risperidone $(n=1)$, or not treated with any antipsychotics $(n=3)$. Despite the differences in delirium etiology for each group, the final K-DRS-98 or MMSE scores were similar 7 days after diagnosis. Other studies reported common underlying etiologies of delirium, such as fracture, metabolic disturbance, or endocrine disturbance $[19,20]$. These inconsistent findings reflect the varying clinical settings from each study, suggesting that the etiologies of delirium need to be investigated in larger samples.

Regarding the pharmacological treatment of delirium, there are few randomized, controlled trials of acute drug treatments [21]. Typical antipsychotics, primarily haloperidol, have been used empirically to control symptoms of delirium. Recently, researchers have begun reporting that atypical antipsychotics, such as olanzapine, quetiapine, and risperidone, might be as effective as haloperidol in delirium treatment $[10,11,18,19]$.

Because of the low incidence of extrapyramidal side effects, quetiapine has been an attractive choice in elderly patients who are more susceptible to such side effects. Compared to haloperidol, patients with delirium on quetiapine showed similar improvement on the DRS and quetiapine was better tolerated [10]. An open-label trial using quetiapine at a mean dosage of $44.9 \pm 31.0 \mathrm{mg} /$ day demonstrated that it was effective and that it improved delirium severity and cognition that were measured by the DRS and MMSE, respectively [22]. Risperidone also showed efficacy in delirium treatment compared with haloperidol treatment in a double-blind trial. The mean daily dose of risperidone was $1.02 \mathrm{mg}$, and the mean daily dose of haloperidol was $1.71 \mathrm{mg}$ [11].

Amisulpride is an atypical antipsychotic used to treat schizophrenia, and it has shown similar effectiveness to olanzapine and risperidone in a large-scale clinical trial [23]. The effectiveness of amisulpride in treating delirium has not been extensively investigated, but one randomized, open prospective study suggests that amisulpride and quetiapine are useful drugs for treating delirium [24]. Results from the present study also support the similar effectiveness of amisulpride to quetiapine and risperidone in treating delirium in elderly patients.

Despite these reports, the usage of antipsychotic medications for delirium treatment remains controversial $[25,26]$. A comprehensive systematic literature review outlined seven high-quality studies: four had decreased delirium rates, five demonstrated no difference in any other clinical outcome examined, and one had worsened clinical outcomes [27]. Cognitive and noncognitive symptoms of delirium could naturally improve [28] through the control of environmental factors [29] or management of underlying etiologies [30]. In the present trial, the data support previous research indicating that there are no benefits to antipsychotic treatment in older patients with delirium. Subjects who were not prescribed any antipsychotic medication showed similar improvement in delirium symptoms 7 days after diagnosis. The demographic data and baseline K-DRS-98 score of the atypical antipsychotic treatment group and non-antipsychotic group showed no difference, which indicates that there was no selection bias between the antipsychotic treatment group and the non-antipsychotic group.

The K-DRS-98 total and severity score of the atypical antipsychotic treatment group and the non-antipsychotic group showed no difference in delirium severity improvement on days 3 and 7 of the clinical course. These findings suggest that antipsychotic treatment did not contribute to early recovery from delirium symptoms compared to the non-antipsychotic group. This finding differs from a previous study of 36 patients who were randomly assigned to quetiapine or placebo treatment, concluding that delirium resolved faster in patients who received quetiapine [31]. This difference might result from the differing patient population and the setting of the intensive care unit versus the general ward. Other trials comparing the treatment response of atypical antipsychotics based on age ( $<70$ years, $\geq 70$ years) suggest that increasing age predict a poor response to delirium treatment in spite of antipsychotic treatment $[32,33]$. Our data were collected from inpatients aged $>65$ years, and the mean age of enrolled subjects was $76.2 \pm 6.6$ years. Data from the present study support the assertion that increased age predicts poor response to antipsychotic treatment for delirium.

Antipsychotics are an obvious choice for treating delirium because many of its symptoms are similar to those of psychoses. Antipsychotic treatment of delirium is based on the hypothesis that excessive dopaminergic activity alone, and combined with acetylcholine, plays an important role in delirium. In the present study, no subscale of the K-DRS-98 showed a difference in improvement between the atypical antipsychotic treatment group and the non-antipsychotic group, including sleep-wake cycle, perceptions and hallucinations, delusions, lability of affect, language, thought process abnormalities, motor agitation, motor retardation, orientation, attention, short-term memory, long-term memory, and visuospatial ability. It should be noted that the pathophysiology of delirium associated with critical illness remains largely uncharacterized and may vary depending on the cause. Some researchers have suggested that the GABAergic and cholinergic neurotransmitter systems play a contributory role because of the increased risk associated with the use of GABAA agonists and anticholinergic drugs. In particular, the central cholinergic deficiency may be a final common pathway. An alternative hypothesis holds that the direct neurotoxic effects of inflammatory cytokines might have contributed to the results found in the present research. These hypotheses remain unproven, making pharmacological management strategies largely empirical.

Because delirium is primarily a cognitive disorder, bedside assessment of cognition is critical to proper diagnosis. All cognitive domains are affected in delirium, including orientation, attention, short-term and long-term memory, visuoconstructive ability, and executive function; however, attention deficits are the most specifically emphasized in DSM. The MMSE score in the atypical antipsychotic treatment group and the non-antipsychotic group showed no 
difference at baseline, and similar improvement on days 3 and 7 following diagnosis. It is of note that the final MMSE score was $14.3 \pm$ 7.3 for all groups, including the non-antipsychotic group. This finding demonstrates that subjects remained severely impaired in cognitive function 7 days after diagnosis in this study.

The results from the present study suggest that there are unclear benefits of antipsychotic treatment in elderly people with delirium. Common side effects of antipsychotic use among the elderly include anticholinergic activity and alpha-receptor blockade, such as confusion, cognitive and functional decline, sedation, hypotension, orthostasis, dizziness, falls, urinary incontinence, voiding problems, and increased risk of urinary infections [34]. In elderly subjects, adverse events include increased risk of death, stroke, extrapyramidal symptoms, and urinary symptoms [35]. Because of the side effects, further studies are needed to clarify the benefits of antipsychotic medications in treating delirium, especially in older subjects.

\section{Limitations}

This study has several limitations. First, the use of a clinician's empirical judgment to assign antipsychotic medications without randomization might be a source of bias and limit our findings. However, the authors found no significant difference in demographic and clinical characteristics among the four groups. Second, the study population, which included patients with both medical and surgical illnesses, was heterogeneous. This feature could be a major limiting factor in the interpretation of our findings even though there were no significant differences in the clinical characteristics of the four groups. Finally, because the present study was designed to recruit patients who were referred to a consultation-liaison psychiatric service, the patient cohort might be skewed to include active psychiatric symptoms.

The atypical antipsychotic treatment group and non-antipsychotic group showed similar improvement in delirium severity and cognitive impairment 7 days after diagnosis. The present study suggests that antipsychotic treatment of delirium might not be efficacious in subjects aged $>65$ years. Non-pharmacological treatment should be considered as the first line of treatment in delirious elderly patients. Nonetheless, there remains a need for the acute management of delirium symptoms, such as agitation and behavioral issues, which might be harmful to the patient and their caregivers. Pharmacological treatment for delirium requires further research into its underlying etiology and pathophysiology.

\section{Acknowledgements}

The authors declare no conflict of interest.

\section{References}

1. Inouye SK, van Dyck CH, Alessi CA, Balkin S, Siegal AP, et al. (1990) Clarifying confusion: the confusion assessment method. A new method for detection of delirium. Ann Intern Med 113: 941-948.

2. Fong TG, Tulebaev SR, Inouye SK (2009) Delirium in elderly adults: Diagnosis, prevention and treatment. Nat Rev Neurol 5: 210-220.

3. Inouye SK (1998) Predisposing and precipitating factors for delirium in hospitalized older patients. Dement Geriatr Cogn Disord 10: 393-400.

4. Bucht G, Gustafson Y, Sandberg O (1999) Epidemiology of delirium. Dementia and geriatric cognitive disorders 10: 315-318.

5. Witlox J, Eurelings LS, de Jonghe JF, Kalisvaart KJ, Eikelenboom P, et al (2010) Delirium in elderly patients and the risk of postdischarge mortality, institutionalization, and dementia: A meta-analysis. JAMA 304: 443-451.

6. Skrobik Y (2011) Delirium prevention and treatment. Anesthesiology Clinics 29: 721-727.
7. Hshieh TT, Fong TG, Marcantonio ER, Inouye SK (2008) Cholinergic deficiency hypothesis in delirium: a synthesis of current evidence. J Gerontol A Biol Sci Med Sci 63: 764-772.

8. Lacasse $H$, Perreault MM, Williamson DR (2006) Systematic review of antipsychotics for the treatment of hospital-associated delirium in medically or surgically ill patients. Ann Pharmacother 40: 1966-1973.

9. Tune $L$ (2002) The role of antipsychotics in treating delirium. Curr Psychiatry Rep 4: 209-212.

10. Maneeton B, Maneeton N, Srisurapanont M, Chittawatanarat K (2013) Quetiapine versus haloperidol in the treatment of delirium: a double-blind, randomized, controlled trial. Drug Des Devel Ther 7: 657

11. Han CS, Kim YK (2004) A double-blind trial of risperidone and haloperidol for the treatment of delirium. Psychosomatics 45: 297-301.

12. American Psychiatric Association (2000) Diagnostic and statistical manual-text revision (DSM-IV-TRim, 2000).

13. Levenson JL (2011) The American psychiatric publishing textbook of psychosomatic medicine: Psychiatric care of the medically ill. American Psychiatric Pub.

14. Trzepacz PT, Mittal D, Torres R, Kanary K, Norton J, et al. (2001) Validation of the delirium rating scale-revised-98: Comparison with the delirium rating scale and the cognitive test for delirium. J Neuropsychiatry Clin Neurosci 13: 229242

15. Lim KO, Kim SY, Lee YH, Lee SW, Kim JL (2006) A validation study for the Korean version of Delirium Rating Scale-Revised-98 (K-DRS-98). Journal of Korean Neuropsychiatric Association 45: 518-526.

16. Folstein MF, Folstein SE, McHugh PR (1975) "Mini-mental state". A practica method for grading the cognitive state of patients for the clinician. J Psychiatr Res 12: 189-198.

17. Woods SW (2003) Chlorpromazine equivalent doses for the newer atypical antipsychotics. Journal of Clinical Psychiatry.

18. Skrobik YK, Bergeron N, Dumont M, Gottfried SB (2004) Olanzapine vs haloperidol: Treating delirium in a critical care setting. Intensive Care Med 30: 444-449.

19. Grover S, Kumar V, Chakrabarti S (2011) Comparative efficacy study of haloperidol, olanzapine and risperidone in delirium. J Psychosom Res 71: 277 281.

20. Grover S, Kate N, Agarwal M, Mattoo SK, Avasthi A, et al. (2012) Delirium in elderly people: A study of a psychiatric liaison service in north India. Int Psychogeriatr 24: 117-127.

21. Bourne RS, Tahir TA, Borthwick M, Sampson EL (2008) Drug treatment of delirium: Past, present and future. J Psychosom Res 65: 273-282.

22. Sasaki Y, Matsuyama T, Inoue S, Sunami T, Inoue T, et al. (2003) A prospective, open-label, flexible-dose study of quetiapine in the treatment of delirium. J Clin Psychiatry 64: 1316-1321.

23. Pani L, Villagran JM, Kontaxakis VP, Alptekin K (2008) Practical issues with amisulpride in the management of patients with schizophrenia. Clin Drug Investig 28: 465-477.

24. Lee KU, Won WY, Lee HK, Kweon YS, Lee CT, et al. (2005) Amisulpride versus quetiapine for the treatment of delirium: a randomized, open prospective study. Int Clin Psychopharmacol 20: 311-314.

25. Boettger S, Breitbart W (2005) A typical antipsychotics in the management of delirium: A review of the empirical literature. Palliative \& supportive care 3: 227237

26. Peritogiannis V, Stefanou E, Lixouriotis C, Gkogkos C, Rizos DV (2009) Atypical antipsychotics in the treatment of delirium. Psychiatry Clin Neurosci 63: 623-631.

27. Trzepacz P, Breitbart W, Franklin J, Levenson J, Martini RD, et al. (1999) Practice guideline for the treatment of patients with delirium. Am J Psychiatry 156: $1-20$

28. Tahir TA, Eeles E, Karapareddy V, Muthuvelu P, Chapple S, et al. (2010) A randomized controlled trial of quetiapine versus placebo in the treatment of delirium. J Psychosom Res 69: 485-490.

29. Lundström M, Edlund A, Karlsson S, Brännström B, Bucht G, et al. (2005) A 
Citation: Lee M, Kim T, Ahn S, Kim JL (2017) Effectiveness of Antipsychotics on Delirium in Elderly Patients. J Psychiatry 20: 411. doi:10.4172/23785756.1000411

multifactorial intervention program reduces the duration of delirium, length of hospitalization, and mortality in delirious patients. J Am Geriatr Soc 53: 622628.

30. Ozbolt LB, Paniagua MA, Kaiser RM (2008) A typical antipsychotics for the treatment of delirious elders. J Am Med Dir Assoc 9: 18-28.

31. Devlin JW, Roberts RJ, Fong JJ, Skrobik Y, Riker RR, et al. (2010) Efficacy and safety of quetiapine in critically ill patients with delirium: a prospective multicenter, randomized, double-blind, placebo-controlled pilot study. Crit Care Med 38: 419-427.

32. Breitbart W, Tremblay A, Gibson C (2002) An open trial of olanzapine for the treatment of delirium in hospitalized cancer patients. Psychosomatics 43: 175182.
33. Kim SW, Yoo JA, Lee SY, Kim SY, Bae KY, et al. (2010) Risperidone versus olanzapine for the treatment of delirium. Hum Psychopharmacol 25: 298-302.

34. Muench J, Hamer AM (2010) Adverse effects of antipsychotic medications. Am Fam Physician 81: 617-622.

35. Maher AR, Maglione M, Bagley S, Suttorp M, Hu JH, et al. (2011) Efficacy and comparative effectiveness of atypical antipsychotic medications for off-label uses in adults: A systematic review and meta-analysis. JAMA 306: 1359-1369. 